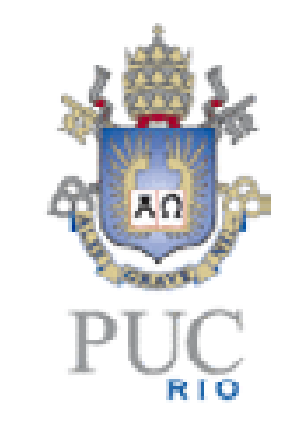

RICARDO DRUMOND DE MOURA

\title{
SELEÇÃO E ORDENAÇÃO DE CONTINGÊNCIAS PARA AVALIAÇÃO DAS CONDIÇÕES DE SEGURANÇA DE TENSÃO
}

\begin{abstract}
Dissertação de Mestrado
Dissertação apresentada como requisito parcial para obtenção do grau de Mestre pelo Programa de Pós-graduação em Engenharia Elétrica do Departamento de Engenharia Elétrica da PUC-Rio.
\end{abstract}

Orientadores: Ricardo Bernardo Prada, Ph.D.

Rio de Janeiro

Outubro de 2002 


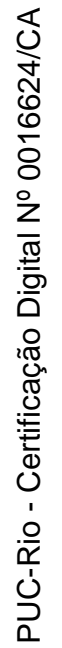


Todos os direitos reservados. É proibida a reprodução total ou parcial do trabalho sem autorização da universidade, do autor e do orientador.

\section{Ricardo Drumond de Moura}

Graduou-se em Engenharia Elétrica, com habilitação em Sistemas de Potência pela Universidade Federal Fluminense em 1979. Atualmente é engenheiro do Departamento de Estudos Elétricos da ELETROBRÁS Centrais Elétricas Brasileiras S.A.

Ficha Catalográfica

Moura, Ricardo Drumond de
Seleção e ordenação de contingências
para avaliação das condições de segurança
de tensão / Ricardo Drumond de Moura;
orientador: Ricardo Bernardo Prada. - Rio de
Janeiro, PUC, Departamento de Engenharia
Elétrica, 2002.
[36], 132 f. : il. ; 30 cm
Dissertação (mestrado) - Pontifícia
Universidade Católica do Rio de Janeiro,
Departamento de Engenharia Elétrica.
Inclui referências bibliográficas.
1. Engenharia Elétrica - Teses. 2.
Estabilidade de tensão. 3. Colapso de tensão.
4. Seleção e ordenação de contingências. I.
Prada, Ricardo Bernardo. II. Pontifícia
Universidade Católica do Rio de Janeiro.
Departamento de Engenharia Elétrica. III.
Título.

CDD: 621.3 


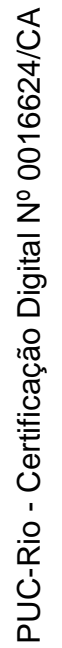




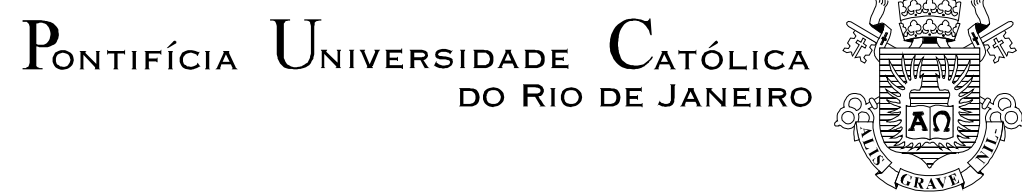

Ricardo Drumond de Moura

\section{Seleção e Ordenação de Contingências \\ para Avaliação das Condições de Segurança de Tensão}

Dissertação apresentada como requisito parcial para obtenção do grau de Mestre pelo Programa de Pós-Graduação em Engenharia Elétrica do Departamento de Engenharia Elétrica do Centro Técnico Científico da PUC-Rio. Aprovada pela Comissão Examinadora abaixo assinada.

Prof. Ricardo Bernardo Prada

Orientador

Departamento de Engenharia Elétrica - PUC-Rio

Prof. Jorgeson Oliveira Rodrigues dos Santos

UFSJ

Prof. Luiz Carlos Pereira da Silva

UNICAMP

Prof. Ney Augusto Dumont

Coordenador Setorial do Centro

Técnico Científico - PUC-Rio

Rio de Janeiro, 28 de outubro de 2002 


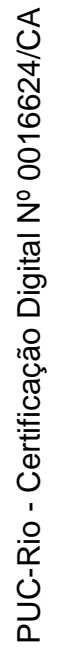


Aos meus queridos pais:

Walter Ferreira de Moura (em memória) e Marlene Drumond de Moura 


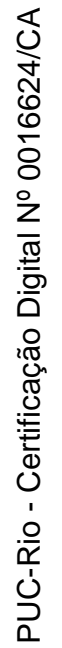




\section{Meus agradecimentos}

a Deus, acima de tudo;

à ELETROBRÁS por me proporcionar a realização deste Curso;

ao Professor Ricardo B. Prada, orientador da dissertação, pelo apoio e pela confiança;

ao Amigo e Professor Leonardo Xavier da Silva, pela inestimável colaboração;

aos Amigos da ELETROBRÁS pelo apoio e incentivo;

à minha querida Esposa Giane, pelo companheirismo, incentivo e paciência; ao meu querido Filho Rodrigo, pela amizade, carinho e incentivo. 


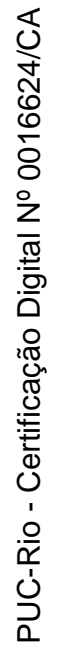




\section{Resumo}

O uso de compensação de potência reativa, embora permita um carregamento mais elevado do sistema elétrico, leva-o a operar próximo de situações de colapso de tensão. Portanto, tornou-se necessário analisar o comportamento do sistema quanto a esse fenômeno na ocorrência de contingências. Neste trabalho alguns métodos existentes são estudados para verificar a capacidade de classificação das contingências que possam afetar o sistema, e como esses métodos procedem para ordená-las por severidade e selecionar aquelas mais prejudiciais. Os métodos são estudados tendo como foco principal a operação em tempo real.

Este trabalho propõe um método capaz de ordenar e selecionar uma lista de contingências prováveis, com base em índices nodais de avaliação das condições de segurança de tensão. Esses índices são baseados na margem em MVA para o máximo carregamento, indicam a região de operação na curva $\mathrm{V} \times \mathrm{P}, \mathrm{Q}$ e a importância relativa entre barras. O Índice de Sensitividade, que indica a redução da margem de potência diante de uma contingência, é estudado minuciosamente. Além da análise nodal, também é proposta uma forma de análise sistêmica, capaz de ordenar e selecionar as contingências de acordo com as influências sobre todo o sistema elétrico.

São apresentados resultados numéricos correspondentes ao sistema Brasileiro Interligado Norte-Nordeste, com carga máxima de junho de 1992. A análise da eficiência computacional do método proposto demonstrou ser possível sua utilização na operação em tempo real integrado à ferramenta de avaliação da segurança de tensão, através de uma estrutura de dados comum aos dois processos.

\section{Palavras-chave}

Estabilidade de tensão, colapso de tensão, seleção e ordenação de contingências. 


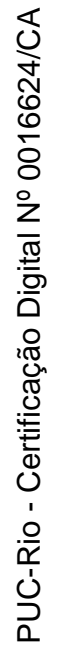




\section{Abstract}

Although the use of compensation of reactive power allows a higher loading in the electric system, it leads it to work closer to voltage collapse situations. Therefore it's necessary to assess the behavior of the system regarding to this phenomena in the occurrence of the contingencies. In this work some existing methods are studied to check the capacity of ranking the contingencies that might affect the system, and how these methods rank them by severity and select those which are more damaging. The methods are studied having as the main focus the real-time operation.

This work proposes a method which is able to rank and select a list of probable contingencies, having as a basis, nodal indexes of voltage security conditions assessment. These indexes are based on MVA margin to the maximum loading, indicate the region of operation on $\mathrm{V} \times \mathrm{P}, \mathrm{Q}$ curve, and the relative importance among buses. The sensitivity index which indicates the reduction of the power margin before a contingency, is studied in detail. Besides the nodal analysis, it is proposed a form of a systemic analysis which is able to rank and select the contingencies according to their influence upon all electrical system.

Numerical results corresponding to the North-Northeast Brazilian Interconected power system, with maximum load of June 1992 are presented. The analysis of computational efficiency of the proposed method showed that it's possible to be used in real-time operation connected to the voltage assessment security tool, through a common structure of data to both procedures.

\section{Keywords}

Voltage stability, voltage collapse, ranking and selecting contingencies. 


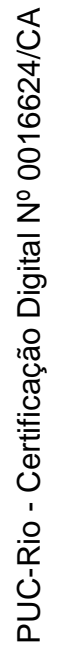




\section{Sumário}

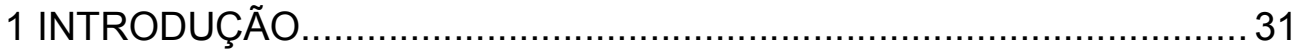

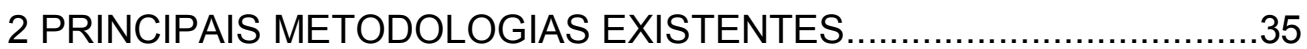

2.1 Método RSI - "Reactive Suport Index" (Índice de Suporte Reativo ) e/ou IF - "Iterative Filtering" (filtragem terativa)................................ 35

2.1.1 RSI - "Reactive Suport Index".................................................. 36

2.1.2 IF - "Iterative Filtering" usando o Método de Correção de Newton 42

2.1.3 IF - "Iterative Filtering" usando o Método da Bisseção................. 51

2.1.3.1 Tratando de uma única contingência....................................... 51

2.1.3.2 Tratando de várias contingências.......................................... 53

2.1.4 Requisitos computacionais............................................... 56

2.1.4.1 RSI - "Reactive Suport Index".......................................... 56

2.1.4.2 IF-"Iterative Filtering" usando o Método de Correção de Newton 56

2.1.4.3 IF-"Iterative Filtering" usando o Método da Bisseção............... 57

2.2 Método utilizado pelo programa VSAT.................................... 58

2.2.1 Técnica computacional principal............................................ 58

2.2.2 Módulo de "Contingency Screening"....................................... 59

2.2.2.1 Índice de severidade para classificação de contingências........ 59

2.2.2.2 IF - "Iterative Filtering"..................................................... 62

3 MÉTODO PROPOSTO PARA SELEÇÃO E ORDENAÇÃO DE CONTINGÊNCIAS

3.1 Índices para a avaliação nodal do carregamento da rede de transmissão........................................................................ 65

3.1.1 Ferramenta Analítica........................................................... 65

3.1.1.1 Magnitude do determinante da matriz $\left[\mathrm{D}^{\prime}\right]$..........................6.67

3.1.1.2 Sinal do determinante da matriz $\left[\mathrm{D}^{\prime}\right]$.................................. 71 


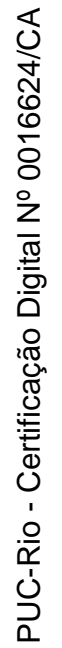




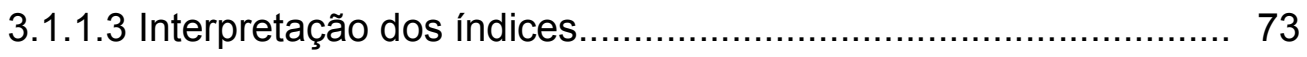

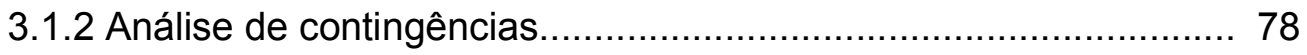

3.2 Seleção e ordenação de contingências - Método proposto............... 79

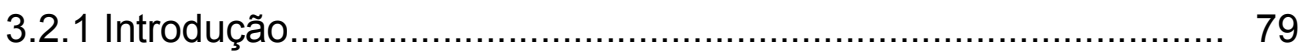

3.2.2 Cálculo das margens do caso base e dos casos contingências.. 79

3.2.3 Seleção e ordenação de contingências através de avaliação

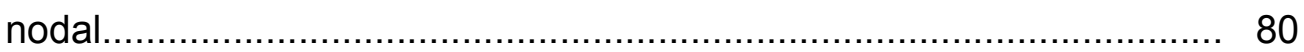

3.2.3.1 Ordenação de contingências utilizando o Índice de

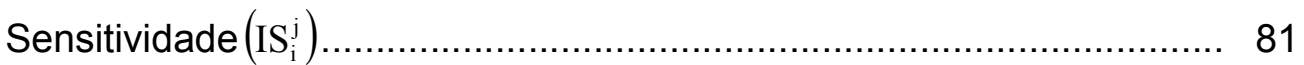

3.2.3.2 Seleção de contingências utilizando o Índice de

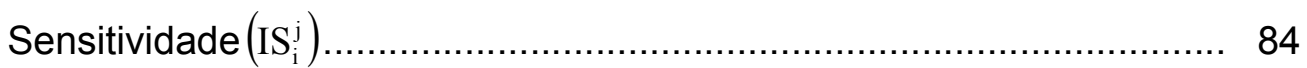

3.2.3.3 Ordem de grandeza do Índice de Sensitividade $\left(\mathrm{IS}_{\mathrm{i}}^{\mathrm{j}}\right)$ para

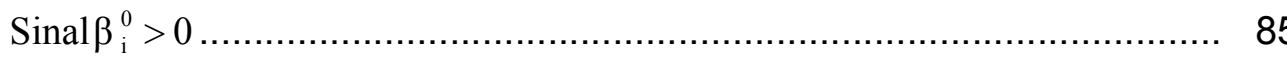

3.2.3.4 Ordem de grandeza do Índice de Sensitividade $\left(\mathrm{IS}_{\mathrm{i}}^{\mathrm{j}}\right)$ para

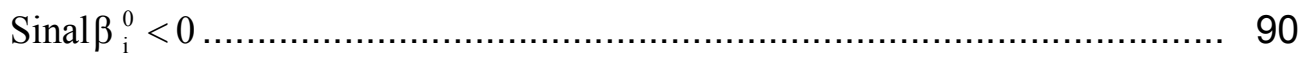

3.2.3.5 Interpretação da escala do $\operatorname{IS}_{\mathrm{i}}^{j}$ de acordo com o

$\operatorname{Sinal} \beta_{i}^{0}$

3.2.4 Seleção e ordenação de contingências através de avaliação de todo o sistema..................................................................... 98

3.2.4.1 Ordenação de contingências utilizando o Índice de Sensitividade para todo o sistema ( sem considerar a importância relativa

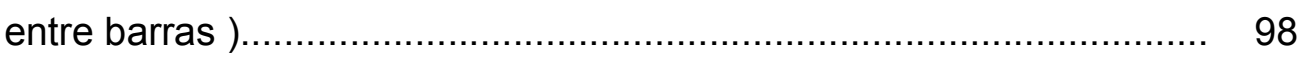

3.2.4.2 Ordenação de contingências utilizando o Índice de Sensitividade para todo o sistema ( considerando a importância relativa entre barras )

3.2.4.3 Seleção de contingências utilizando um Índice Crítico de Sensitividade para todo o sistema $\left(\mathrm{IS}_{\mathrm{s}}^{\mathrm{c}}\right)$ 101

4 CÁLCULO NUMÉRICO 103

4.1 Introdução. 103 


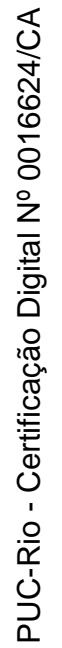


4.2 Ordenação das contingências pelo Índice de Sensitividade do sistema sem considerar a ponderação pelo Índice de Importância das barras

4.3 Ordenação das contingências pelo Índice de Sensitividade do sistema considerando a ponderação pelo Índice de Importância das barras

4.4 Barras de tensão controlada.

4.5 Ordenação das contingências divergentes através do número de iterações

4.6 Contingências não executadas

4.7 Seleção das contingências.

5 CONCLUSÕES

Apêndice A - UNIFILAR do sistema equivalente sul-sudeste do sistema interligado nacional 


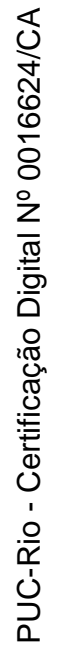




\section{Lista de Tabelas}

Tabela 2.1 Resultados do IF pelo Método de Correção de Newton para o sistema da BC Hydro.

Tabela 2.2 Resultados do IF pelo Método de Correção de Newton para o sistema equivalente sul-sudeste do Sistema Interligado Nacional....... 49

Tabela 2.3 Resultados do IF pelo Método da Bisseção para o sistema equivalente sul-sudeste do Sistema Interligado Nacional.

Tabela 2.4 Critério de classificação por ordem de severidade das contingências convergentes.

Tabela 2.5 Lista resultante do "Iterative Filtering" utilizando o Método da

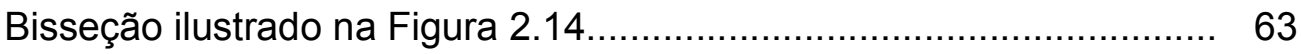

Tabela 4.1 Relatório de saída do ESTABTEN para o caso base ........ 105 Tabela 4.2 Relatório de saída do ESTABTEN para a contingência 28.

Tabela 4.3 Relatório de saída do ESTABTEN para a contingência 218 .

Tabela 4.4 Contingências em ordem de severidade decrescente ordenadas pelo Índice de Sensitividade - $\sum_{\mathrm{i}} \mathrm{IS}_{\mathrm{i}}^{\mathrm{j}}$

Tabela 4.5 Índice de Importância das barras

Tabela 4.6 Contingências em ordem de severidade decrescente ordenadas pelo Índice de Sensitividade $-\sum_{\mathrm{i}} \mathrm{IS}_{\mathrm{i}}^{\mathrm{j}}$ com ponderação pelo

Índice de Importância das barras.

Tabela 4.7 Barras que apresentam ângulo $\beta_{\mathrm{i}}^{\mathrm{j}}<0$ e respectivas contingências

Tabela 4.8 Contingências divergentes em ordem de severidade decrescente ordenadas pelo número de iterações 154

Tabela A-1 Contingências aplicadas ao caso base (sistema equivalente sul-sudeste). 


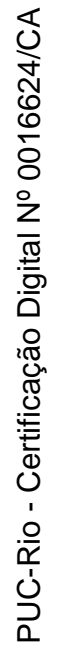




\section{Lista de Figuras}

Figura2.1 $\quad \mathrm{RCI}_{\mathrm{j}}$ - llustração do Índice de Compensação Reativa para a barra j.

Figura 2.2 RCI $\mathrm{j}_{\mathrm{j}}$ - llustração do Índice de Compensação Reativa para a barra ( correção da figura 2.1 ).

Figura 2.3 Ilustração do Índice de Suporte Reativo para a barra j... 39

Figura 2.4 Quantidade extra de potência reativa utilizada pela barra $p$

na pós-contingência

Figura 2.5 Classificação das contingências pelo "Iterative Filtering". 42

Figura 2.6 Rampas utilizadas no "Iterative Filtering" pelo Método de Correção de Newton.

Figura 2.7 Rampas utilizadas no "Iterative Filtering" pelo Método de Correção de Newton.

Figura 2.8 Ilustração do "Iterative Filtering" pelo Método de Correção de Newton. 50

Figura 2.9 "Iterative Filtering" pelo Método da Bisseção para contingência única.

Figura 2.10 "Iterative Filtering" pelo Método da Bisseção aplicado a várias contingências simultaneamente.

Figura 2.11 Obtenção dos níveis de carregamento pelo "Iterative

Filtering" usando o Método da Bisseção.

Figura 2.12 llustração do "Iterative Filtering" pelo Método da Bisseção.

Figura 2.13 Ordenação pelo número de iterações de casos de contingências divergentes aplicadas ao caso base.

Figura 2.14 "Iterative Filtering" pelo Método da Bisseção utilizado pelo VSAT. 62

Figura 3.1 Região de Operação da curva $V \times \mathrm{P}, \mathrm{Q}$. 65

Figura 3.2 Localização do vetor gradiente de $P_{i}$ e $Q_{i}$ no plano $\mathrm{V} \times \theta$ 


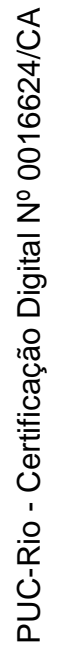


Figura 3.3 Projeção das curvas $\mathrm{P}_{\mathrm{i}}$ constante, $\mathrm{Q}_{\mathrm{i}}$ constante e $\phi_{\mathrm{i}}$ constante nos planos $\mathrm{V} \times \mathrm{P}, \mathrm{Q}$ e $\mathrm{V} \times \mathrm{\theta}$

Figura 3.4 Localização de pontos de operação na curva $\vee \times P, Q$ e suas respectivas margens.

Figura 3.4(a) Ponto de operação localizado na parte superior da curva $V \times P, Q$

Figura 3.4(b) Ponto de operação localizado na parte inferior da curva $V \times P, Q$.

Figura 3.4(c) Ponto de operação localizado na "ponta do nariz" da curva $\mathrm{V} \times \mathrm{P}, \mathrm{Q}$ 76

Figura 3.5 Curva $V \times P, Q$ do caso base e do caso contingência com suas respectivas margens de potência.

Figura 3.6 Curvas do caso base e contingências com suas respectivas margens de potência e Índices de Sensitividade.

Figura 3.7 Caso base e contingências selecionadas pelo Índice de Sensitividade Crítico.

Figura 3.8(a) Ponto de operação localizado na região normal das curvas $V \times P, Q$ do caso base e caso contingência, com $\mathrm{S}_{\mathrm{m}_{\mathrm{i}}}^{\mathrm{j}} \cong \mathrm{S}_{\mathrm{m}_{\mathrm{i}}}^{0}$ 85

Figura 3.8(b) Ponto de operação localizado na região normal das curvas $\mathrm{V} \times \mathrm{P}, \mathrm{Q}$ do caso base e caso contingência, com $\mathrm{S}_{\mathrm{m}_{\mathrm{i}}}^{\mathrm{j}} \cong \mathrm{S}_{\mathrm{i}}^{0} \ldots \ldots \ldots \ldots \ldots . . . \ldots 6$ Figura 3.8(c) Ponto de operação localizado na região normal das curvas $V \times P, Q$ do caso base e na região anormal do caso contingência......

Figura 3.9 Caso base e contingências com seus respectivos limites de Índice de Sensitividade.

Figura 3.10(a) Ponto de operação localizado na região anormal da curva $V \times P, Q$ do caso base e na região anormal do caso contingência com queda de tensão final.

Figura 3.10(b) Ponto de operação localizado na região anormal da curva $V \times P, Q$ do caso base e na região anormal do caso contingência com aumento de tensão final

Figura 3.10(c) Ponto de operação localizado na região anormal da curva $V \times P, Q$ do caso base e na "ponta do nariz" do caso contingência com aumento de tensão final 


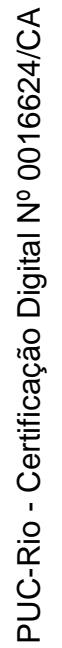


Figura 3.10(d) Ponto de operação localizado na região anormal da curva $V \times P, Q$ do caso base e na região normal de operação do caso contingência com aumento significativo de tensão final...................... 94

Figura 3.11 Caso base e contingências com seus respectivos limites de Índices de Sensitividade.

Figura 3.12 Escala do Grau de Severidade baseado no valor de $\mathrm{IS}_{\mathrm{i}}^{\mathrm{j}}$

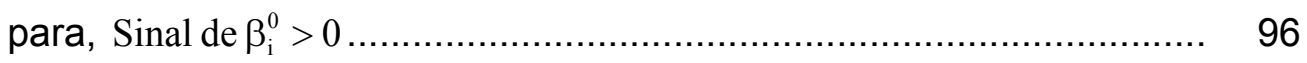

Figura 3.13 Escala do Grau de Severidade baseado no valor de $I S_{i}^{j}$

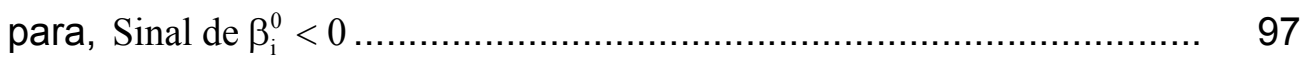
Figura 4.1 Gráfico do Índice de Sensitividade das contingências que convergiram..

Figura 4.2 Gráfico do Índice de Sensitividade das primeiras 100 contingências convergentes. 151

Figura A-1 Caso base: Sistema equivalente sul-sudeste. 167 


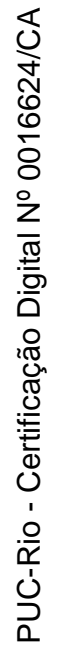




\section{Lista de Abreviaturas}

$\begin{array}{lll}\text { FACTS } & : & \text { Flexible AC Transmission System } \\ \text { RSI } & : & \text { Reactive Suport Index } \\ \text { IF } & : & \text { Iterative Filtering } \\ \text { RCI } & : & \text { Reactive Compensation Index } \\ \text { ULTC } & : & \text { Under Load Tap Changer } \\ \text { SVC } & : & \text { Static var Compensator } \\ \text { BC Hydro } & : & \text { British Columbia's Hydro and Power Authority } \\ \text { VSAT } & : & \text { Voltage Security Assessment Tool } \\ \text { EMS } & : & \text { Energy Management System } \\ \text { FDPF } & : & \text { Fast Decoupled AC Power Flow }\end{array}$




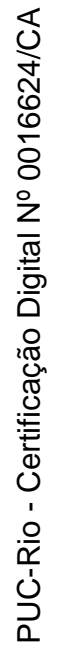

